\title{
ON INSTABILITY OF EXTREMALS OF POTENTIAL ENERGY FUNCTIONAL
}

\author{
N.M. POLUBOYAROVA
}

\begin{abstract}
The paper is devoted to studying the stability and instability of extremals of a potential energy functional. A particular case of this functional is the area type functionals. The potential energy functional is the sum of functionals of area type and of volume density of forces. The potential energy functional is constructed in such way in order to take into consideration the loads on the surface from outside and inside. The stability is defined as the sign-definiteness of the second variation. In this paper we prove the formulae for the first and second variations of the functional. We also prove that the extremal surface can be locally minimal and locally maximal depending on the sign of matrix $G$. Using the $G$-capacity and the second variation of the functional, we obtain the conditions for the instability of the extremals of the potential energy functional. This technique was developed in works by V.M. Miklyukov and V.A. Klyachin. For G-parabolic extremal surfaces we prove the degeneracy into the plane. This result is an analogue of the theorems by M. do Carmo and C.K. Peng. By an example of $n$-dmensional surfaces of revolution we demonstrate the formulae for the first and second variations of the functional. We also prove the criteria of stability and instability for $n$-dimensional surfaces of revolution. Similar extremal surfaces arise in applications, in physical problems (e.g. soap films, capillary surfaces, magnetic liquids in a gravitational field with a potential), and the properties of extreme surfaces are used in applied problems (e.g. modeling of awning coverings).
\end{abstract}

Keywords: the variation of functional, extreme surface, area type functional, volumetric power density functional, functional of potential energy, $G$-capacity, $G$-parabolicity, the stability.

Mathematics Subjects Classifications: 53A10, 30C70, 31A15.

\section{INTRODUCTION}

The present paper is devoted to studying the stability and instability of extremals for a special functional. This is one of topical and challenging issues of the contemporary analysis. Similar extremal surfaces arise in applications, in physical problems (e.g., soap films) and the properties of the extremal surfaces are employed in application problems (e.g., modeling of tent coverings).

The problem of stability of extremal surfaces in various spaces was studied in papers by D. Hoffman and R. Osserman [1], J.L. Barbosa and M. do Carmo [2], J. Lowson [3], A.V. Pogorelov [4], H. B. Simons [5], A.A. Tuzhilin [6], A.T. Fomenko [7] etc.

Extremals of a particular case of the considered functional describe, for example, equilibrium fluids in gravitational field with a potential that was described in the monography by R.Finn. Some particular cases of extremal surfaces with the mean curvature depending only on one

Poluboyarova Natalia Mikhailovna On instability of EXtremals of potential Energy funcTIONAL.

(c) Poluboyarova N.M. 2017.

The study is supported by RFBR (grant no. 15-41-02479_r).

Submitted June 20, 2017. 
fixed coordinate were considered in the paper of D. Hoffman and R. Osserman [1]. In the paper [9], V.A. Klyachin the studied the stability of surfaces with a prescribed mean curvature.

In the paper the potential energy functional is represented as a linear combination of arealike functional and functional with volume density of forces. This is motivated by the need of considering inward and outward loads on a surface (system) in applied problems. This is why in the present work we consider a more complicated functional than the area-like one. The results are new and more general as compared to the classical facts known for minimal surfaces; and there are no restrictions for the mean curvature of the surfaces. In the proofs we employ the capacity technique elaborated by V.M. Mikljukova and V.A. Klyachin in papers [10] that allow us to prove analogous tests for the new functional.

\section{MAin Results}

Let $M$ be a $n$-dimensional connected oriented $C^{2}$-manifold. We define a potential energy functional on the oriented hypersurface $\mathcal{M}=(M, u)$ obtained by $C^{2}$-immersion $u: M \rightarrow \mathbb{R}^{n+1}$ :

$$
W(\mathcal{M})=\int_{\mathcal{M}} \Phi(\xi) d \mathcal{M}+\int_{\Omega} \Psi(x) d x
$$

where $\Omega \subset \mathbb{R}^{n+1}$ is a domain such that $\mathcal{M} \subset \partial \Omega$, and, from a physical point of view, $\Psi(x)$ is the volume density of forces acting on a fluid element occupying the interior of the domain $\Omega$, and $\Phi, \Psi: \mathbb{R}^{n+1} \rightarrow \mathbb{R}$ are $C^{2}$-smooth functions and $\xi$ is the field of unit normals to the surface $\mathcal{M}$.

Let $V$ be a $C^{2}$-smooth vector field defined in a neighbourhood of the surface $\mathcal{M}$ such that $\left.V\right|_{\mathcal{M}}=h \cdot \xi$ where $h \in C_{0}^{1}(\mathcal{M}), \xi$ is the field of unit normals to the surface, and it is supposed that the integral curves of the field $V$ are located on the straight lines and the identity $|V|=$ const holds along these curves.

It is clear that provided the surface $\mathcal{M}$ is immersed, each vector field $V=h \cdot \xi$ defined along $\mathcal{M}$ can be continued into some neighbourhood of $\mathcal{M}$ so that the above conditions are satisfied. We note that according work [5], the second variation is independent of choice of the continuation.

Let $U(\mathcal{M})$ be a neighbourhood of the surface $\mathcal{M}$, in which the field $V$ is defined, and $g_{t}(x): U(\mathcal{M}) \rightarrow \mathbb{R}^{n+1}$ be a one-parameter group of local diffeomorphisms generated by the vector field $V$. That is, $g_{t}(x)$ is a solution to the Cauchy problem:

$$
\frac{d g_{t}(x)}{d t}=V\left(g_{t}(x)\right),\left.\quad g_{t}(x)\right|_{t=0}=x .
$$

We let $\mathcal{M}_{t}=g_{t}(\mathcal{M})$. It is clear that $\mathcal{M}_{0}=\mathcal{M}$.

The surface $\mathcal{M}$ is extremal if the first variation of functional (1) vanishes for all infinitesimal deformations of the surface $\mathcal{M}$. Extremal surface $\mathcal{M}$ is stable if the second variation of functional (1) is sign-definite for all infinitesimal deformations of the surface $\mathcal{M}$; otherwise it is called unstable.

A Riemmanian metrics and a corresponding scalar product of tangent vectors are induced on the surface $\mathcal{M}$. This scalar product will be denoted in the same way as the in $\mathbb{R}^{n+1}$, by $\langle\cdot, \cdot\rangle$. The symbols $\bar{\nabla}$ and $\nabla$ stand for the Riemannian connectivities in $\mathbb{R}^{n+1}$ and $\mathcal{M}$, respectively. The following relations are known

$$
\nabla h=(\bar{\nabla} h)^{T}, \quad \nabla_{X} Y=\left(\bar{\nabla}_{X} Y\right)^{T},
$$

which are valid for all $C^{1}$-smooth functions $h: \mathbb{R}^{n+1} \rightarrow \mathbb{R}$ and $C^{1}$-smooth vector fields $X$ and all $Y$ tangential to $\mathcal{M}$. By symbol $v^{T}$ we denote the orthogonal projection of a vector $v$ on the tangent plane $T_{m} \mathcal{M}$ of the surface $\mathcal{M}$ at a point $m \in \mathcal{M}$. 
We denote

$$
G=\left\{G_{i j}\right\}_{i, j=1}^{n+1}, G_{i j}=\frac{\partial^{2} \Phi}{\partial \xi_{i} \partial \xi_{j}}+\delta_{i j}(\Phi-\langle D \Phi, \xi\rangle)
$$

where $D \Phi=\left(\frac{\partial \Phi}{\partial \xi_{1}}, \frac{\partial \Phi}{\partial \xi_{2}}, \ldots, \frac{\partial \Phi}{\partial \xi_{n+1}}\right), \delta_{i j}$ is the Kronecker delta, $k_{i}$ are the principle curvatures; and $E_{i}$ are the principle directions of the surface.

Theorem 1. If $W(t)=W\left(\mathcal{M}_{t}\right)$, then

$$
W^{\prime}(0)=\int_{\mathcal{M}}\left(\operatorname{div}(D \Phi(\xi))^{T}-n H \Phi(\xi)+\Psi(x)\right) h(x) d \mathcal{M}
$$

where div is divergence in the metrics on the surface $\mathcal{M}$ and $H=\langle\vec{H}, \xi\rangle$ is the mean curvature of the surface $\mathcal{M}$ with respect to the normal $\xi$. Moreover, if $W^{\prime}(0)=0$ for all functions $h(x) \in C_{0}^{1}(\mathcal{M})$, then

$$
W^{\prime \prime}(0)=\int_{\mathcal{M}}\left\{G(\nabla h, \nabla h)+h^{2}\left(\langle\bar{\nabla} \Psi(x), \xi\rangle-\sum_{i=1}^{n} k_{i}^{2} G\left(E_{i}, E_{i}\right)\right)\right\} d \mathcal{M}
$$

where $G$ is the quadratic form associated with matrix (2).

Functional (1) is the composition of the above studied area-like functionals [11 and the functional with volumetric density of forces [9].

Hereafter we suppose that the matrix $G$ is positively definite and $\langle\bar{\nabla} \Psi, \xi\rangle \leqslant 0$.

Remark. The restrictions for the matrix $G$ are motivated by using of capacity concept in the proofs and by employing in applications. The condition $\langle\bar{\nabla} \Psi, \xi\rangle \leqslant 0$ is in fact a coupling one between inwards and outwards surface loads but its interpretation in applications is not known to the author. The presence of conditions is due to features of the technique of the proof as in paper [9].

Corollary 1. If the matrix $G$ is positively definite, then each extremal is locally-minimal for functional (1), and if the matrix $G$ is negatively definite, all extremals are locally-maximal.

Let $\Omega_{1} \subset \mathcal{M}$ be a domain in the surface $\mathcal{M}$ and $P, Q \subset \Omega_{1}$ be two disjoint closed sets in $\bar{\Omega}_{1}$. Every triple $\left(P, Q ; \Omega_{1}\right)$ will be referred to as the capacitor on $\mathcal{M}$.

The $G$-capacity of a capacitor $\left(P, Q ; \Omega_{1}\right)$ is the quantity

$$
\operatorname{cap}_{G}\left(P, Q ; \Omega_{1}\right)=\inf _{\varphi} \int_{\mathcal{M}} G(\nabla \varphi, \nabla \varphi) d \mathcal{M}
$$

where the infimum is taken over all locally Lipschitz functions $\varphi: \mathcal{M} \rightarrow \mathbb{R}^{1}, \varphi(m)=1$ as $m \in P$ and $\varphi(m)=0$ as $m \in Q$.

The surface $\mathcal{M}$ will be referred to as $G$-parabolic if there exists a sequence of subdomains $\Omega_{k} \subset \mathcal{M}, \Omega_{k} \Subset \Omega_{k+1}$ such that the identity

$$
\lim _{k \rightarrow \infty} \operatorname{cap}_{G}\left(P, \partial \Omega_{k} ; \Omega_{k}\right)=0
$$

holds for each $P \Subset \mathcal{M}$.

Theorem 2. Let $\mathcal{M}$ be an extremal surface for functional (1). If there exists a domain $\Omega_{1} \subset \mathcal{M}$ and a compact set $P \Subset \Omega_{1}$ such that

$$
\int_{P}\left(\sum_{i=1}^{n} k_{i}^{2} G\left(E_{i}, E_{i}\right)\right)>\operatorname{cap}_{G}\left(P, \partial \Omega_{1} ; \Omega_{1}\right)
$$

then the surface $\mathcal{M}$ is unstable. 
Theorem 3. Let $\mathcal{M}$ be a stable extrinsically complete complete (without edge) immersed surface extremal for the functional (1) along which the inequality $\langle\bar{\nabla} \Psi, \xi\rangle \leqslant 0$ holds. If $\mathcal{M}$ is of a $G$-parabolic type, then the surface $\mathcal{M}$ is a plane.

Remark. The theorem 3 is an analogue of known result due to M. do Carmo and C.K. Peng [12].

\section{ProOFs}

Proof of Theorem 1. The proof is reduced to a combination of previously obtained results. In order to use them, we represent the functional (1) in the form

$$
W(\mathcal{M})=F(\mathcal{M})+L(\mathcal{M})
$$

where

$$
\begin{aligned}
& F(\mathcal{M})=\int_{\mathcal{M}} \Phi(\xi) d \mathcal{M} \\
& L(\mathcal{M})=\int_{\Omega} \Psi(x) d x .
\end{aligned}
$$

The following theorem on the variations of functional (3) was proved by B.A. Klyachin and the author in [11].

Theorem 4. If $F(t)=F\left(\mathcal{M}_{t}\right)$, then

$$
F^{\prime}(t)=\int_{\mathcal{M}}\left(\operatorname{div}(D \Phi(\xi))^{T}-n H \Phi(\xi)\right) h(x) d \mathcal{M} .
$$

Moreover, if $F^{\prime}(0)=0$, then

$$
F^{\prime \prime}(0) \equiv \int_{\mathcal{M}}\left\{G(\nabla h, \nabla h)-h^{2} \sum_{i=1}^{n} k_{i}^{2} G\left(E_{i}, E_{i}\right)\right\} d \mathcal{M}
$$

holds for all functions $h \in C_{0}^{1}(\mathcal{M})$.

Theorem 5 for the functional (4) was proved in [9] while studying another functional.

Theorem 5. If $L(t)=L\left(\mathcal{M}_{t}\right)$, then

$$
L^{\prime}(t)=\int_{\mathcal{M}} \Psi(x) h(x) d \mathcal{M} .
$$

Moreover, if $L^{\prime}(0)=0$, then

$$
L^{\prime \prime}(0)=\int_{\mathcal{M}}(\langle\bar{\nabla} \Psi, \xi\rangle-n H \Psi(x)) h^{2} d \mathcal{M}
$$

holds for all functions $h(x) \in C_{0}^{1}(\mathcal{M})$.

It is obvious that $W^{\prime}(t)=F^{\prime}(t)+L^{\prime}(t), W^{\prime \prime}(0)=F^{\prime \prime}(0)+L^{\prime \prime}(0)$. The proof is complete.

Proceeding to the proof of Corollary 1, we note that it is based on a method developed in paper [13]. 
Proof of Corollary 1. By Theorem 1 we have that for extremal surfaces the identity $W^{\prime}(0)=0$ holds and the second variation is well-defined. We fix a point $p \in \mathcal{M}$ and a neighbourhood $\mathcal{U} \subset \mathcal{M}$ of the point $p$. we let

$$
c=\sup _{\mathcal{U}}\left(\sum_{i=1}^{n} k_{i}^{2} G\left(E_{i}, E_{i}\right)-\langle\bar{\nabla} \Psi(x), \xi\rangle\right) .
$$

For an arbitrary function $h \in C_{0}^{1}(\mathcal{U})$ we have

$$
\begin{aligned}
Q(h) & \equiv \int_{\mathcal{M}}\left\{G(\nabla h, \nabla h)-h^{2}\left(\sum_{i=1}^{n} k_{i}^{2} G\left(E_{i}, E_{i}\right)-\langle\bar{\nabla} \Psi(x), \xi\rangle\right)\right\} d \mathcal{M} \\
& \geqslant \int_{\mathcal{M}}\left\{G(\nabla h, \nabla h)-h^{2} c\right\} d \mathcal{M} \geqslant \int_{\mathcal{M}}\left\{\inf _{h_{0} \in C_{0}^{1}(\mathcal{U})} \frac{\int \mathcal{U} G\left(\nabla h_{0}, \nabla h_{0}\right)}{\int_{\mathcal{U}} h_{0}^{2}}-c\right\} h^{2} d \mathcal{M} .
\end{aligned}
$$

If the matrix $G$ is positive-definite, we can state that

$$
\inf _{h_{0} \in C_{0}^{1}(\mathcal{U})} \frac{\int_{\mathcal{U}} G\left(\nabla h_{0}, \nabla h_{0}\right)}{\int_{\mathcal{U}} h_{0}^{2}} \rightarrow+\infty
$$

as $\operatorname{diam} \mathcal{U} \rightarrow 0$, and then it is obvious that $Q(h) \geqslant 0$ for a sufficiently small neighbourhood $\mathcal{U}$ of the point $p$. Hence, the variation of the surface $\mathcal{M}$ along the direction of the vector field $\xi$ does not lessen the area and the surface $\mathcal{M}$ is locally-minimal.

Arguing in the same way for a negative-definite matrix $G$, we observe that

$$
\inf _{h_{0} \in C_{0}^{1}(\mathcal{U})} \frac{\int_{\mathcal{U}} G\left(\nabla h_{0}, \nabla h_{0}\right)}{\int_{\mathcal{U}} h_{0}^{2}} \rightarrow-\infty
$$

as diam $\mathcal{U} \rightarrow 0$ and as above, we conclude that $Q(h) \leqslant 0$ if the support of the function $h$ is sufficiently small. Therefore, the variation of the surface $\mathcal{M}$ does not lessen the area and the surface $\mathcal{M}$ is locally-maximal. The proof is complete.

The proof is based on the following lemma proved in [11].

Lemma. Assume that we are given a non-zero non-negative function $S(x)$ in $\mathbb{R}^{n+1}$. Then for an arbitrary bounded domain $\Omega_{1} \subset \mathcal{M}$ and a compact set $P \Subset \Omega_{1}$ there exists a function $h_{0}: \mathcal{M} \rightarrow \mathbb{R}$ such that $h_{0} \in C_{0}^{1}\left(\Omega_{1}\right)$ obeying

$$
Q_{S}\left(h_{0}\right)=\int_{\mathcal{M}}\left\{G\left(\nabla h_{0}, \nabla h_{0}\right)-S h_{0}^{2}\right\} d \mathcal{M} \leqslant \operatorname{cap}_{G}\left(P, \partial \Omega_{1} ; \Omega_{1}\right)-\int_{P} S d \mathcal{M} .
$$

Proof of Theorem 2. We apply Lemma to the the second variation of functional and in view of the condition $\langle\bar{\nabla} \Psi, \xi\rangle \leqslant 0$ we obtain the inequality

$$
\begin{aligned}
\int_{\mathcal{M}}\{G(\nabla h, \nabla h) & \left.+h^{2}\left(\langle\bar{\nabla} \Psi(x), \xi\rangle-\sum_{i=1}^{n} k_{i}^{2} G\left(E_{i}, E_{i}\right)\right)\right\} d \mathcal{M} \\
& \leqslant \int_{\mathcal{M}}\left\{G(\nabla h, \nabla h)-h^{2} \sum_{i=1}^{n} k_{i}^{2} G\left(E_{i}, E_{i}\right)\right\} d \mathcal{M} \\
& \leqslant \operatorname{cap}_{G}\left(P, \partial \Omega_{1} ; \Omega_{1}\right)-\int_{P}\left(\sum_{i=1}^{n} k_{i}^{2} G\left(E_{i}, E_{i}\right)\right) d \mathcal{M} .
\end{aligned}
$$


And since by the assumption of the theorem, the $G$-capacity is less than the last term in the above inequality, we get that the second variation is negative. By the definition, this means that the surface $\mathcal{M}$ is unstable. The proof is complete.

Proof of Theorem 3. The proof is similar to that provided in [11] for functional (3).

Let $P \subset \mathcal{M}$ be an arbitrary compact set. Provided the surface $\mathcal{M}$ of $G$-parabolic type, there exists a subsequence of domains $\Omega_{k} \subset \mathcal{M}, \Omega_{k} \subset \Omega_{k+1}$ and $P \Subset \Omega_{1} \subset \Omega_{2} \ldots$, such that the identity holds:

$$
\lim _{k \rightarrow \infty} \operatorname{cap}_{G}\left(P, \partial \Omega_{k} ; \Omega_{k}\right)=0 .
$$

Since by the assumptions of the theorem $\mathcal{M}$ is a stable extremal surface, the definiton of stability implies that the second variation is nonnegative:

$$
0 \leqslant \int_{\mathcal{M}}\left\{G(\nabla h, \nabla h)-h^{2}\left(\sum_{i=1}^{n} k_{i}^{2} G\left(E_{i}, E_{i}\right)-\langle\bar{\nabla} \Psi(x), \xi\rangle\right)\right\} d \mathcal{M} .
$$

Employing the condition $\langle\bar{\nabla} \Psi, \xi\rangle \leqslant 0$, we get

$$
0 \leqslant \int_{\mathcal{M}}\left\{G(\nabla h, \nabla h)-h^{2} \sum_{i=1}^{n} k_{i}^{2} G\left(E_{i}, E_{i}\right)\right\} d \mathcal{M} .
$$

It is known [14 that the variational problem

$$
\int_{\mathcal{M}} G(\nabla h, \nabla h) d \mathcal{M} \rightarrow \inf
$$

has solutions $h_{k}(x)$ for all $k=1,2, \ldots$, such that

$$
\operatorname{cap}_{G}\left(P, \partial \Omega_{k} ; \Omega_{k}\right)=\int_{\mathcal{M}} G\left(\nabla h_{k}, \nabla h_{k}\right) d \mathcal{M}
$$

and $\left.h_{k}\right|_{P}=1,\left.\quad h_{k}\right|_{\partial \Omega_{k}}=0$. Then we get

$$
\begin{aligned}
0 & \leqslant \int_{\mathcal{M}}\left\{G\left(\nabla h_{k}, \nabla h_{k}\right)-h_{k}^{2}\left(\sum_{i=1}^{n} k_{i}^{2} G\left(E_{i}, E_{i}\right)\right)\right\} d \mathcal{M} \\
& =\operatorname{cap}_{G}\left(P, \partial \Omega_{k} ; \Omega_{k}\right)-\int_{\mathcal{M}} h_{k}^{2}\left(\sum_{i=1}^{n} k_{i}^{2} G\left(E_{i}, E_{i}\right)\right) d \mathcal{M} \\
& \leqslant \operatorname{cap}_{G}\left(P, \partial \Omega_{k} ; \Omega_{k}\right)-\int_{P}\left(\sum_{i=1}^{n} k_{i}^{2} G\left(E_{i}, E_{i}\right)\right) d \mathcal{M} .
\end{aligned}
$$

This implies that

$$
\begin{aligned}
& 0 \leqslant \operatorname{cap}_{G}\left(P, \partial \Omega_{k} ; \Omega_{k}\right)-\int_{P}\left(\sum_{i=1}^{n} k_{i}^{2} G\left(E_{i}, E_{i}\right)\right) d \mathcal{M} \\
& \operatorname{cap}_{G}\left(P, \partial \Omega_{k} ; \Omega_{k}\right) \geqslant \int_{P}\left(\sum_{i=1}^{n} k_{i}^{2} G\left(E_{i}, E_{i}\right)\right) d \mathcal{M} .
\end{aligned}
$$


Passing to the limit as $k \rightarrow+\infty$ in this inequality and using the definition of $G$-parabolicity of the surface, we get:

$$
\int_{P}\left(\sum_{i=1}^{n} k_{i}^{2} G\left(E_{i}, E_{i}\right)\right) d \mathcal{M} \leqslant 0,
$$

and this is possible only as $\sum_{i=1}^{n} k_{i}^{2} G\left(E_{i}, E_{i}\right) \equiv 0$ on $P$. Since the compact set $P$ was chosen arbitrarily, we conclude that $\sum_{i=1}^{n} k_{i}^{2} G\left(E_{i}, E_{i}\right) \equiv 0$ on $\mathcal{M}$.

Since the matrix $G$ is the positive-definite, this means that for all $i$ the identities $k_{i}=0$ are true, where $k_{i}$ are the principle curvatures of the surface $\mathcal{M}$. This means that $\mathcal{M}$ is a plane.

\section{Examples of Realisations of Obtained Results}

Let $\mathcal{M} \subset \mathbb{R}^{n+1}$ be a $C^{2}$-smooth surface defined by the position vector

$$
\vec{R}(t, \theta)=(t, r(t) \rho(\theta)),
$$

$\theta \in \mathbb{S}^{n-1}, \quad \rho(\theta)$ be the position vector of the sphere $\mathbb{S}^{n-1}, t \in(a, b) \subset \mathbb{R}$; and let $r(t)$ be a $C^{2}$-smooth function on $(a, b), \xi_{n+1}$ be the coordinate of unit normal to the surface $\mathcal{M}$ and $\Phi(\xi)=\phi\left(\xi_{n+1}\right), \Psi(x)=\psi\left(x_{n+1}\right)=\psi(t)$.

We introduce the following notations to have more compact statements

$$
\begin{aligned}
& \tau=\xi_{n+1}=-\dot{r}(t) / \sqrt{1+\dot{r}^{2}(t)}, \\
& \phi^{\prime}(\tau)=d \phi / d \xi_{n+1}, \phi^{\prime \prime}(\tau)=d^{2} \phi / d \xi_{n+1}^{2}, \\
& \dot{r}(t)=d r(t) / d t, \ddot{r}(t)=d^{2} r(t) / d t^{2}, \\
& B(t)=\frac{\phi^{\prime \prime}(\tau)}{\left(1+\dot{r}^{2}(t)\right)\left(\phi(\tau)+\frac{\phi^{\prime}(\tau) \dot{r}(t)}{\sqrt{1+\dot{r}^{2}(t)}}\right)}, \\
& C(t)=\frac{r(t) \sqrt{1+\dot{r}^{2}(t)}}{\phi(\tau)+\frac{\phi^{\prime}(\tau) \dot{r}(t)}{\sqrt{1+\dot{r}^{2}(t)}}}, \\
& Q(t)=\phi(\tau)+\frac{\phi^{\prime}(\tau) \dot{r}(t)}{\sqrt{1+\dot{r}^{2}(t)}} .
\end{aligned}
$$

Then Theorem 1 implies Theorem 6 on equation of extremals (the proof is given in [15]) and Theorem 7 on expression of the second variation for the revolution surface.

Theorem 6. The surface $\mathcal{M}$ of class $C^{2}$ defined by the radius-vector (5) is extremal for the functional (1) if and only if

$$
\frac{r(t) \ddot{r}(t)}{1+\dot{r}^{2}(t)}-\frac{(n-1)+\Psi(x) C(t)}{B(t)+1}=0 .
$$

The following theorem is a logical continuation of Theorem 6 and it is useful in seeking stability and instability domains of extremal revolution surfaces.

Theorem 7. An extremal surface $\mathcal{M}$ of the class $C^{2}$ defined by position-vector (5) is stable if and only if the quadratic form

$$
\int_{\mathcal{M}} h_{t}^{\prime 2}(t, \theta) \frac{\phi^{\prime \prime}(\tau)+Q(t)\left(1+\dot{r}^{2}(t)\right)}{\left(1+\dot{r}^{2}(t)\right)^{2}}+\left|D_{\theta} h(t, \theta)\right|^{2} \frac{Q(t)}{r^{2}(t)}
$$




$$
-h^{2}(t, \theta) \cdot\left(\frac{\left((n-1) Q(t)+\psi(t) r(t) \sqrt{1+\dot{r}^{2}(t)}\right)^{2}}{r^{2}(t)\left(\phi^{\prime \prime}(\tau)+Q(t)\left(1+\dot{r}^{2}(t)\right)\right)}+\frac{(n-1) Q(t)}{r^{2}(t)\left(1+\dot{r}^{2}(t)\right)}+\frac{\psi^{\prime}(t) \dot{r}(t)}{\sqrt{1+\dot{r}^{2}(t)}}\right) d \mathcal{M}
$$

is sign-definite over the class of all Lipschitz functions $h: \mathbb{R}^{n+1} \rightarrow \mathbb{R}$ such that $h \in C_{0}^{1}(\mathcal{M})$.

Proof of Theorem 7. All intermediate steps in calculating of the coefficients in quadratic form, principle curvatures and principle directions of the surface were described in details in [15] and this is why we do not provide them. To calculate the second variation, we should write out the obtained formulae for the first and second quadratic form and substitute them into the formula for the second variation; we also going to clarify how to obtain the values of the matrix $G$ on the vectors $E_{i}$ and $\nabla h$.

The first quadratic form is

$$
I=\left(1+\dot{r}^{2}(t)\right) d t^{2}+r^{2}(t) d \theta^{2}
$$

where $d \theta^{2}$ is the length differential for $\mathbb{S}^{n-1}$.

The second quadratic form is

$$
I I=\frac{\ddot{r}(t)}{\sqrt{1+\dot{r}^{2}(t)}} d t^{2}+\frac{r(t)}{\sqrt{1+\dot{r}^{2}(t)}} d \widetilde{\theta}^{2}
$$

where $d \widetilde{\theta}^{2}$ is the second quadratic form for $\mathbb{S}^{n-1}$.

Using the standard notation $\left\|g_{i j}\right\|$ for matrix of the coefficients of the first quadratic form and the notation $\left\|g^{i j}\right\|$ for the inverse matrix, we write out the formula for gradient of the function $h$ :

$$
(\nabla h)^{i}=\sum_{j=0}^{n-1} g^{i j} \frac{\partial h}{\partial x_{j}}, \quad i=\overline{0, n-1}
$$

and we calculate $h$ :

$$
\begin{aligned}
(\nabla h)^{0} & =\sum_{j=0}^{n-1} g^{0 j} \frac{\partial h}{\partial x_{j}}=g^{00} \frac{\partial h}{\partial x_{0}}=\frac{h_{t}^{\prime}}{1+\dot{r}^{2}(t)} \\
(\nabla h)^{i} & =\sum_{j=0}^{n-1} g^{i j} \frac{\partial h}{\partial x_{j}}=\sum_{j=1}^{n-1} g^{i j} \frac{\partial h}{\partial x_{j}}=\frac{1}{r^{2}(t)} \sum_{j=1}^{n-1} \tilde{g}^{i j} \frac{\partial h}{\partial \theta_{i j}}=\frac{1}{r^{2}(t)}(D h)^{i}, \quad i \neq 0 .
\end{aligned}
$$

Denoting $D_{\theta} h=\sum_{i=1}^{n-1}(D h)^{i}$, we obtain coordinate expression for the gradient vector:

$$
\nabla h=\left(\frac{h_{t}^{\prime}}{1+\dot{r}^{2}(t)}, \frac{D_{\theta} h}{r^{2}(t)}\right)
$$

This easily implies that the square of the modulus of the gradient is of the form

$$
|\nabla h|^{2}=h_{t}^{\prime 2} /\left(1+\dot{r}^{2}(t)\right)+\left|D_{\theta} h\right|^{2} / r^{2}(t)
$$

because $|\nabla h|^{2}=\sum_{j=0}^{n-1} g_{i j}\left((\nabla h)^{i}\right)^{2}$. The values of the matrix $G$ on the vectors $E_{i}$ and $\nabla h$ are calculated by means of the scalar product $\left\langle\left\langle G, E_{i}\right\rangle, E_{i}\right\rangle,\langle\langle G, \nabla h\rangle, \nabla h\rangle$ and are written as follows:

$$
\begin{aligned}
& G\left(E_{1}, E_{1}\right)=\frac{\phi^{\prime \prime}(\tau)}{1+\dot{r}^{2}(t)}+\phi(\tau)+\frac{\phi^{\prime}(\tau) \dot{r}(t)}{\sqrt{1+\dot{r}^{2}(t)}}, \quad G\left(E_{i}, E_{i}\right)=\phi(\tau)+\frac{\phi^{\prime}(\tau) \dot{r}(t)}{\sqrt{1+\dot{r}^{2}(t)}}, \\
& G(\nabla h, \nabla h)=\frac{\phi^{\prime \prime}(\tau) h_{t}^{\prime 2}}{\left(1+\dot{r}^{2}(t)\right)^{2}}+\left(\frac{h_{t}^{\prime 2}}{1+\dot{r}^{2}(t)}+\frac{\left|D_{\theta} h\right|^{2}}{r^{2}(t)}\right)\left(\phi(\tau)+\frac{\phi^{\prime}(\tau) \dot{r}(t)}{\sqrt{1+\dot{r}^{2}(t)}}\right) .
\end{aligned}
$$


We substitute the obtained expressions into the formula for the second variation in Theorem 1 and this gives us the required statement. The proof is complete.

\section{BIBLIOGRAPHY}

1. D. Hoffman, R. Osserman. The area of generalized gaussian image and the stability of minimal surfaces in $S^{n}$ and $\mathbb{R}^{n} / /$ Math. Ann. 260:4, 437-452 (1982).

2. J.L. Barbosa, M. do Carmo. Stability of minimal surfaces and eigevalues of the Laplacian // Math. Z. 173:1, 13-28 (1980).

3. H.B. Lawson. Some intrinsic characterizations of minimal surfaces // J. Anal. Math. 24:1, 151161 (1971).

4. A.V. Pogorelov. On stability of minimal surfaces // Dokl. Akad. Nauk SSSR. 260:2, 293-295 (1981). [Sov. Math. Dokl. 24, 274-276 (1981).]

5. J. Simons. Minimal varieties in riemannian manifolds // Ann. Math. 88:1, 62-105 (1968).

6. A.A. Tuzhilin, A.T. Fomenko. Elements of the geometry of minimal surfaces in three-dimensional space. Nauka, Moscow (1991). [Transl. Math. Monog. 93. Amer. Math. Soc., Providence, RI (1991).]

7. A.T. Fomenko. On growth rate and smallest volumes of globally minimal surfaces in cobordisms // Trudy Semin. Vect. Tenz. Anal. Moscow State Univ. 21, 3-12 (1985). (in Russian).

8. R. Finn. Equilibrium capillary surfaces. Springer, New York (1986).

9. V.A. Klyachin. On some properties of stable and unstable surfaces with prescribed mean curvature // Izv. RAN. Ser. Matem. 70:4, 77-90 (2006). [Izv. Math. 70:4, 77-90 (2006)].

10. V.A. Klyachin, V.M. Miklyukov. Criteria of instability of surfaces of zero mean curvature in warped Lorentz products // Matem. Sborn. 187:11, 67-88 (1996). [Sb. Math. 187:11, 1643-1663 (1996).]

11. V.A. Klyachin, N.M. Medvedeva. On stability of some functionals of area type // Sibir. Electron. Matem. Izv. 4, 113-132 (2007).

12. M. do Carmo, C.K. Peng. The stable minimal surfaces in $\mathrm{R}^{3}$ are planes // Bull. (Ne Ser.) Amer. Math. Soc. 1:6, 903-906 (1979).

13. V.A. Klyachin, V.M. Miklyukov. Maximal tubular hypersurfaces in Minkowsky space // Izv. AN SSSR. 55:1, 206-217 (1992). [ Math. USSR Izv. 38:1, 203-213 (1992).]

14. V.M. Gol'dstein, Yu.G. Reshetniak. Quasiconformal mappings and Sobolev spaces. Nauka, Moscow (1983). [Math. Appl. Soviet Series. 54. Kluwer Academic Publ., Dordrecht.]

15. N.M. Poluboyarova. Equations of extremals of potential energy // Vestn. Volgograd. Gosud. Univ. Ser. 1. Matem. Fiz. 5(36), 60-72 (2016). (In Russian)

Natalia Mikhailovna Poluboyarova,

Volgograd State University,

University avenue, 100,

400062, Volgograd, Russia

E-mail: natasha_medvedeva@volsu.ru 